\title{
Higher Derivative Corrections in Holographic QCD
}

\author{
Anirban Basu 1 \\ Institute for Advanced Study, Princeton, NJ 08540, USA
}

\begin{abstract}
We consider the effect of the $\mathcal{R}^{4}$ term in type IIA string theory on the supergravity background dual to $N_{c}$ D4 branes compactified on a circle with supersymmetry breaking boundary conditions. We study the dynamics of $\mathrm{D} 8$ branes in this perturbed geometry in the probe approximation. This leads to an analysis of higher derivative corrections in holographic QCD beyond the supergravity approximation. We make a rough estimate of the corrections to the masses of some of the lightest (axial) vector mesons. The corrections are suppressed by a factor of $\left(g_{Y M}^{2} N_{c}\right)^{-3}$ compared to their supergravity values. We find that the masses of these mesons increase from their supergravity values.
\end{abstract}

\footnotetext{
${ }^{1}$ email: abasu@ias.edu
} 


\section{Introduction}

It is a challenging problem to understand strong coupling phenomena such as confinement and chiral symmetry breaking in QCD at low energies. Using the gauge/string duality, a model of the holographic dual of pure QCD without matter was proposed in [1]. Witten considered $N_{c}$ D4 branes with one of the world volume directions compactified on a circle, with anti-periodic boundary conditions for the fermions. This configuration breaks supersymmetry, and the fermions and the scalars on the world volume theory of the D4 branes become massive at tree level and at one-loop level respectively. Thus at low energies, this reduces to a theory of pure Yang-Mills in four dimensions which is confining. Flavor was added to this model in the probe approximation [2], by which one means that $N_{f}$ flavor branes are placed in the background geometry dual to $N_{c}$ color branes, such that $N_{f} \ll N_{c}$. More recently, Sakai and Sugimoto considered the dynamics of flavor D8 branes in the background geometry of color D4 branes in the probe approximation $[3,4]$. This gives a model of holographic QCD with matter, which differs from QCD at energies comparable to the Kaluza-Klein mass scale of the theory, which is determined by the radius of the circle on which the D4 branes are compactified. Also the theory has an $S O(5)$ symmetry transverse to the color branes, unlike QCD. Nevertheless, this theory is an interesting model in trying to understand QCD at energy scales below the Kaluza-Klein mass scale. Sakai and Sugimoto demonstrated chiral symmetry breaking in this theory by analyzing the dynamics of the flavor branes in the background geometry of the color branes in the supergravity approximation. For related work, see [5-9]. Letting the radius of the circle on which the D4 branes are compactified to go to infinity, one obtains a theory with broken chiral symmetry, but which is unconfined. Such systems have been studied in [10-15] (also see [16] for a related discussion).

Now understanding various aspects of holographic QCD has been so far done at the level of supergravity. In this paper, we attempt to go beyond the supergravity approximation, and include the effects of stringy corrections. In particular, we shall include the effect of the $\mathcal{R}^{4}$ term in the effective action of type IIA string theory. When considering only the supergravity contributions, we are working in the large $N_{c}$ limit, with $g_{Y M}^{2} N_{c} \rightarrow \infty$, where $g_{Y M}$ is the four dimensional gauge coupling. When we consider the contribution due to the $\mathcal{R}^{4}$ term, which is $\alpha^{\prime 3}$ suppressed compared to the supergravity contributions, we consider the leading correction to supergravity where $g_{Y M}^{2} N_{c}$ is kept large but finite.

We first consider the effect of the $\mathcal{R}^{4}$ term on the supergravity background dual to the D4 branes. We analyze the perturbed geometry, and consider the dynamics of the D8 branes 
in this geometry, still in the probe approximation. In particular, we focus on the dynamics of the gauge fields on the world volume theory of the D8 branes. The fluctuations which are along the $(3+1)$ directions are massive, and are interpreted as (axial) vector mesons [3] in holographic QCD. Using the perturbed geometry, we make a very rough estimate of the corrections to the masses of the lightest mesons, due to the higher derivative corrections. At the level of approximation we use, we find that the masses increase from their supergravity values, with $\delta m^{2} \sim\left(g_{Y M}^{2} N_{c}\right)^{-3}$. The analysis of the perturbed geometry is a generalization of the method in $[17,18]$ to non-conformal cases, where the effect of the $\mathcal{R}^{4}$ term on the near-horizon geometry dual to D3 branes in type IIB string theory was considered (also see [19]). We shall see that the analysis gets considerably more complicated, essentially because of the loss of conformality. Though we obtain the exact perturbed geometry, we can solve for the coefficients in the various expressions only recursively as we shall demonstrate below. Coupled with the fact that the mesons masses can only be calculated roughly at the supergravity level, the complexity of the equations allows us to obtain a rather rough estimate of the corrections to the meson masses.

It should be noted that our analysis does not give the complete answer due to the $\alpha^{\prime 3}$ corrections to supergravity. We consider only the $\mathcal{R}^{4}$ term in the entire supermultiplet at $O\left(\alpha^{\prime 3}\right)$, and there are many other terms in this supermultiplet that will also contribute (for example, $\mathcal{R}^{3} F_{4}^{2}, \mathcal{R}^{3}(\partial \phi)^{2}$, and so on $\left.{ }^{2}\right)$. The entire $\mathcal{R}^{4}$ supermultiplet is not well understood (see $[20,21]$ for a relevant discussion in type IIB string theory), and so we restrict ourselves to the $\mathcal{R}^{4}$ term only. Adding the other contributions, it is possible that the values of various observables like meson masses will change.

\section{The background geometry dual to the color branes}

In order to study holographic QCD, we consider $N_{c}$ D4 branes extending along the directions $0,1,2$, and 3 , and compactified along the direction $x_{4}$, with antiperiodic boundary conditions for the fermions to break supersymmetry. The number of colors $N_{c}$ is taken to be very large in the entire discussion.

The dual supergravity background in the string frame is given by 3

$$
d s_{\text {string }}^{2}=\left(\frac{U}{R}\right)^{3 / 2}\left(\eta_{\mu \nu} d x^{\mu} d x^{\nu}+f(U) d x_{4}^{2}\right)+\left(\frac{R}{U}\right)^{3 / 2}\left(\frac{d U^{2}}{f(U)}+U^{2} d \Omega_{4}^{2}\right),
$$

\footnotetext{
${ }^{2} F_{4}$ and $\phi$ are the $\mathrm{R}-\mathrm{R}$ four form and the dilaton respectively.

${ }^{3}$ This is obtained from the near extremal black 4-brane solution [22] by interchanging the role of the time and $x_{4}$ coordinates.
} 
where

$$
e^{\phi}=g_{s}\left(\frac{U}{R}\right)^{3 / 4}, \quad R^{3}=\pi g_{s} N_{c} \alpha^{\prime 3 / 2}, \quad f(U)=1-\frac{U_{K K}^{3}}{U^{3}},
$$

and $U$ is the radial coordinate transverse to the 4 -brane world volume. The four form field strength is given by $F_{4}=Q \omega_{4}$, where $\omega_{4}$ is the volume form on the unit four sphere. Using the Dirac quantization condition [23],

$$
\int_{S^{4}} F_{4}=\frac{\kappa_{10} N_{c}}{\sqrt{\pi \alpha^{\prime}}}
$$

we get that

$$
Q=3 N_{c} \pi \alpha^{\prime 3 / 2}
$$

Including the $\mathcal{R}^{4}$ correction to the supergravity action, the relevant action in the string frame is given by $[24-27] 4$

$$
S_{\text {string }}=\frac{1}{2 \kappa_{10}^{2}} \int d^{10} x \sqrt{-g}\left[e^{-2 \phi}\left(R+4(\nabla \phi)^{2}+\gamma W\right)-\frac{1}{2.4 !} F_{4}^{2}\right]
$$

where

$$
\gamma=\frac{\zeta(3)}{8} \alpha^{\prime 3}
$$

and

$$
W=C^{H M N K} C_{P M N Q} C_{H}^{R S P} C_{R S K}^{Q}+\frac{1}{2} C^{H K M N} C_{P Q M N} C_{H}^{R S P} C_{R S K}^{Q},
$$

where $C^{H}{ }_{M N K}$ is the Weyl tensor. We now want to compute the perturbed background due to the introduction of the $\mathcal{R}^{4}$ interaction. To do so, we find it convenient to go to the Einstein frame which gives the action

$$
S_{\text {Einstein }}=\frac{1}{2 \kappa^{2}} \int d^{10} x \sqrt{-\hat{g}}\left[\hat{R}-\frac{1}{2}(\hat{\nabla} \phi)^{2}-g_{s}^{3 / 2} \frac{e^{\phi / 2}}{2.4 !} \hat{F}_{4}^{2}+g_{s}^{3 / 2} \gamma e^{-3 \phi / 2} \hat{W}\right],
$$

where $\hat{g}$ is the Einstein frame metric, and $\kappa=\kappa_{10} g_{s}$. The metric $\hat{g}_{M N}$ is given by

$$
d s_{\text {Einstein }}^{2}=\left(\frac{U}{R}\right)^{9 / 8}\left(\eta_{\mu \nu} d x^{\mu} d x^{\nu}+f(U) d x_{4}^{2}\right)+\left(\frac{R}{U}\right)^{15 / 8}\left(\frac{d U^{2}}{f(U)}+U^{2} d \Omega_{4}^{2}\right) .
$$

We make the ansatz for the perturbed metric

\footnotetext{
${ }^{4}$ See [28-30] for the analysis using conformal sigma model techniques.
} 


$$
d s_{\text {Einstein }}^{2}=H^{2}(U)\left[K^{2}(U) d x_{4}^{2}+P^{2}(U) d U^{2}+\eta_{\mu \nu} d x^{\mu} d x^{\nu}\right]+L^{2}(U) d \Omega_{4}^{2} .
$$

Thus translational invariance along the $(3+1)$ directions and the $x_{4}$ direction, as well as the transverse $S O(5)$ rotational invariance is preserved. We now want to construct the perturbed metric and the dilaton in the Einstein frame to leading order in $\gamma$. One might think that $F_{4}$ gets perturbed to $F_{4}=Q \lambda(U) \omega_{4}$, where $\lambda(U)=1+O(\gamma)$. However, the Dirac quantization condition (3) prevents this and $F_{4}$ remains unperturbed.

Using the symmetries of the ansatz (10), we see that the action (8) is given by

$$
\begin{array}{r}
S_{\text {Einstein }}=V \int_{U_{K K}}^{\infty} d U \sqrt{\tilde{\hat{g}}}\left[\hat{R}-\frac{1}{2}(\hat{\nabla} \phi)^{2}-\frac{g_{s}^{2}}{2 \cdot 4 !}\left(\frac{U}{R}\right)^{3 / 8} e^{\left(\phi_{1}+\phi_{2}+\ldots\right) / 2} \hat{F}_{4}^{2}\right. \\
\left.+\gamma\left(\frac{U}{R}\right)^{-9 / 8} e^{-3\left(\phi_{1}+\phi_{2}+\ldots\right) / 2} \hat{W}+\right],
\end{array}
$$

where

$$
\sqrt{\tilde{\hat{g}}}=H^{6} K P L^{4}
$$

and

$$
V=\frac{\operatorname{Vol}\left(\mathrm{S}^{4}\right) V_{3,1} \operatorname{Vol}\left(\mathrm{S}^{1}\right)}{2 \kappa^{2}} .
$$

In (13), $\operatorname{Vol}\left(\mathrm{S}^{4}\right)=8 \pi^{2} / 3$ is the volume of the unit four sphere, and $\operatorname{Vol}\left(\mathrm{S}^{1}\right)$ is the circumference of the circle along $x_{4}$. Also we have expressed

$$
\phi=\phi_{0}+\frac{3}{4} \ln \left(\frac{U}{R}\right)+\phi_{1}+\phi_{2}+\ldots,
$$

where $g_{s}=e^{\phi_{0}}, \phi_{1} \sim O(\gamma), \phi_{2} \sim O\left(\gamma^{2}\right)$, and the remaining terms in .. are of $O\left(\gamma^{3}\right)$. Thus in (11), we have that

$$
(\hat{\nabla} \phi)^{2}=\frac{1}{H^{2} P^{2}}\left(\frac{9}{16 U^{2}}+\frac{3}{2 U}\left(\partial_{U} \phi_{1}\right)+\left(\partial_{U} \phi_{1}\right)^{2}+\frac{3}{2 U}\left(\partial_{U} \phi_{2}\right)+O\left(\gamma^{3}\right)\right) .
$$

We describe the various details for obtaining the perturbed metric and the dilaton in the appendices. Note that even though we do not have closed form expressions for the metric or the dilaton perturbations in (78) or (90), we see that these perturbations take a simple form: they are given by sums of positive integer powers of harmonic functions (upto a factor of $\left.U^{-3 / 2}\right)$. This is similar to what happens in the conformal case of the 3 -brane geometry $[17,18]$. 


\section{The flavor branes in the background geometry}

Having obtained the perturbed background dual to the color D4 branes, we now analyze the dynamics of $N_{f}$ probe D8 branes in this background geometry. We work in the probe approximation so that $N_{f} \ll N_{c}$. Before taking the dynamics into account, the $\mathrm{D}$ brane configuration is given by

$\begin{array}{ccccccccccr} & 0 & 1 & 2 & 3 & 4 & 5 & 6 & 7 & 8 & 9 \\ \mathrm{D} 4 \quad: \quad \mathrm{x} & \mathrm{x} & \mathrm{x} & \mathrm{x} & \mathrm{x} & & & & & \\ \mathrm{D} 8, \overline{\mathrm{D} 8}: & \mathrm{x} & \mathrm{x} & \mathrm{x} & \mathrm{x} & & \mathrm{x} & \mathrm{x} & \mathrm{x} & \mathrm{x} & \mathrm{x}\end{array}$

Thus the flavor D8 and $\overline{\mathrm{D} 8}$ branes intersect the color D4 branes along $(3+1)$ dimensions and are separated along the $x_{4}$ direction. This configuration becomes very different when the dynamics are considered [3]. The D8 and $\overline{\mathrm{D} 8}$ branes get connected which is interpreted as chiral symmetry breaking. Our aim is to consider some aspects of the dynamics of the flavor branes in the perturbed background geometry dual to the color branes. First let us see how the flavor branes deform in this background geometry. The background metric is given by

$$
\begin{aligned}
d s_{\text {string }}^{2}= & \left(\frac{U}{R}\right)^{3 / 2}\left[e^{\gamma \hat{\phi}_{1} / 2} \eta_{\mu \nu} d x^{\mu} d x^{\nu}+f(U) e^{\gamma\left(\hat{\phi}_{1} / 2+2 a_{1}+18 b_{1}\right)} d x_{4}^{2}\right] \\
& +\left(\frac{R}{U}\right)^{3 / 2}\left[e^{\gamma\left(\hat{\phi}_{1} / 2+2 b_{1}\right)} \frac{d U^{2}}{f(U)}+U^{2} e^{\gamma\left(\hat{\phi}_{1} / 2+2 c_{1}\right)} d \Omega_{4}^{2}\right] .
\end{aligned}
$$

Considering probe D8 branes in this geometry where $U=U\left(x_{4}\right)$, we see that the induced metric on the D8 brane world volume is given by

$$
\begin{aligned}
d s_{D 8}^{2}= & {\left[\left(\frac{U}{R}\right)^{3 / 2} f(U) e^{\gamma\left(\hat{\phi}_{1} / 2+2 a_{1}+18 b_{1}\right)}+\left(\frac{R}{U}\right)^{3 / 2} e^{\gamma\left(\hat{\phi}_{1} / 2+2 b_{1}\right)} \frac{U^{\prime 2}}{f(U)}\right] d x_{4}^{2} } \\
& +\left(\frac{U}{R}\right)^{3 / 2} e^{\gamma \hat{\phi}_{1} / 2} \eta_{\mu \nu} d x^{\mu} d x^{\nu}+\left(\frac{R}{U}\right)^{3 / 2} U^{2} e^{\gamma\left(\hat{\phi}_{1} / 2+2 c_{1}\right)} d \Omega_{4}^{2},
\end{aligned}
$$

where $U^{\prime}=\left(\partial U / \partial x_{4}\right)$. In the expressions (16) and (17) and in the ones that follow, the exponentials are only to be expanded to $O(\gamma)$. Inserting the induced metric (17) into the DBI action of the D8 branes, we get that 


$$
S_{D 8} \sim \int d^{4} x d x_{4} e^{2 \gamma\left(\hat{\phi}_{1} / 2+2 c_{1}\right)(U)} U^{4} \sqrt{e^{\gamma\left(\hat{\phi}_{1} / 2+2 a_{1}+18 b_{1}\right)(U)} f(U)+\left(\frac{R}{U}\right)^{3} e^{\gamma\left(\hat{\phi}_{1} / 2+2 b_{1}\right)(U)} \frac{U^{\prime 2}}{f(U)}}
$$

Following Sakai and Sugimoto, we look for a solution to the classical equation of motion for the D8 brane resulting from (18) which asymptotes as $U \rightarrow \infty$ to a fixed value of $x_{4}$. This is the position of the flavor brane along the $x_{4}$ direction in the naive picture. To consider a configuration of D8 and $\overline{\mathrm{D} 8}$ branes which connect together leading to chiral symmetry breaking, we also want this configuration to satisfy $U\left(x_{4}=0\right)=U_{0}, U^{\prime}\left(x_{4}=0\right)=0$, such that it is symmetric about $x_{4}=0$. Thus naively, without considering the dynamics, this corresponds to D8 and $\overline{\mathrm{D} 8}$ branes placed symmetrically about $x_{4}=0$. Including the dynamics, they get connected. This solution which has the interpretation of a wormhole solution connecting the D8 and $\overline{\mathrm{D} 8}$ branes asymptotically exhibits chiral symmetry breaking in holographic QCD. The throat of this wormhole has its minimum radius $U_{0}$ at $x_{4}=0$. This solution is given by

$$
x_{4}(U)=U_{0}^{4} \sqrt{\frac{f\left(U_{0}\right)}{\Theta_{2}\left(U_{0}\right)}} \int_{U_{0}}^{U} d U \frac{e^{\gamma\left(\hat{\phi}_{1} / 2+2 b_{1}\right)(U) / 2}}{f(U)\left(\frac{U}{R}\right)^{3 / 2} \sqrt{U^{8} f(U) \frac{\Theta_{1}(U)}{\left.\Theta_{1}\left(U_{0}\right)\right)}-U_{0}^{8} f\left(U_{0}\right) \frac{\Theta_{2}(U)}{\Theta_{2}\left(U_{0}\right)}}},
$$

where

$$
\Theta_{1}(U)=e^{2 \gamma\left(3 \hat{\phi}_{1} / 2+2 a_{1}+18 b_{1}+4 c_{1}\right)(U)}, \quad \Theta_{2}(U)=e^{\gamma\left(\hat{\phi}_{1} / 2+2 a_{1}+18 b_{1}\right)(U)} .
$$

Thus chiral symmetry continues to be broken in the presence of the higher derivative corrections. In (19), $U_{0}$ is an arbitrary parameter satisfying $U_{0} \geq U_{K K}$. For the sake of simplicity, we shall analyze the spectrum for the case $U_{0}=U_{K K}$ as done by Sakai and Sugimoto. Taking only the supergravity action into account, they showed that the D8 and $\overline{\mathrm{D} 8}$ branes are placed at antipodal points on the circle parametrized by $x_{4}$ when $U_{0}=U_{K K}$. Now even when the higher derivative corrections are turned on, the ansatz we have made for the various perturbations preserves the same symmetries, in particular, translational invariance along the $x_{4}$ circle is preserved. Thus when $U_{0}=U_{K K}$, we expect the branes to remain at antipodal points on the $x_{4}$ circle. We now show this is the case.

Given the perturbed metric (16), in order to avoid a conical singularity at $U=U_{K K}$, $x_{4}$ must be a periodic variable satisfying 


$$
x_{4} \sim x_{4}+\frac{4 \pi R^{3 / 2} e^{-\gamma\left(a_{1}+8 b_{1}\right)\left(U_{K K}\right)}}{3 \sqrt{U_{K K}}} .
$$

Thus the circumference of the $x_{4}$ circle is given by

$$
\delta x_{4}=\frac{4 \pi R^{3 / 2} e^{-\gamma\left(a_{1}+8 b_{1}\right)\left(U_{K K}\right)}}{3 \sqrt{U_{K K}}} .
$$

From (19), we can calculate the position in $x_{4}$ where the D8 brane is placed which is given by $x_{4}(\infty)$, and the D8 and $\overline{\mathrm{D} 8}$ branes are separated by twice this distance. However, for $U_{0}=U_{K K}, f\left(U_{0}\right)=0$, and the integrand in (19) diverges at $U=U_{0}$. Thus the expression for $x_{4}(\infty)$ needs to be regularized. We regularize it by setting $\sigma=\left(U_{0} / U_{K K}\right)=1+\epsilon$, and taking the limit $\epsilon \rightarrow 0$. We need to pick out the $O(1 / \sqrt{\epsilon})$ terms from the integral which cancel the $O(\sqrt{\epsilon})$ term from $\sqrt{f\left(U_{0}\right)}$. We have that

$$
\left.x_{4}(\infty)\right|_{U_{0}=U_{K K}}=\frac{R^{3 / 2}}{\sqrt{U_{K K}}} \sqrt{\frac{g(\sigma)}{\Theta_{2}(\sigma)}} \int_{\sigma=1+\epsilon}^{\infty} d u \frac{e^{\gamma\left(\hat{\phi}_{1} / 2+2 b_{1}\right)(u) / 2}}{g(u) u^{3 / 2} \sqrt{u^{8} g(u) \frac{\Theta_{1}(u)}{\Theta_{1}(\sigma)}-\sigma^{8} g(\sigma) \frac{\Theta_{2}(u)}{\Theta_{2}(\sigma)}}},
$$

where

$$
g(u)=1-\frac{1}{u^{3}}
$$

It is straightforward to show that the relevant contribution is contained in the expression given by

$$
\begin{aligned}
\left.x_{4}(\infty)\right|_{U_{0}=U_{K K}} & =\frac{R^{3 / 2}}{3 \sqrt{U_{K K}}} \sqrt{\frac{3 \epsilon}{\Theta_{2}(1)}} \int_{3 \epsilon}^{1} d x \frac{e^{\gamma\left(\hat{\phi}_{1} / 2+2 b_{1}\right)(1) / 2}}{x^{3 / 2}}\left(1-\frac{3 \epsilon}{x}\right)^{-1 / 2} \\
& =\frac{R^{3 / 2}}{3 \sqrt{U_{K K}}} \sqrt{\frac{e^{\gamma\left(\hat{\phi}_{1} / 2+2 b_{1}\right)(1)}}{\Theta_{2}(1)}} \sum_{k=0}^{\infty} \frac{\Gamma(k+1 / 2)}{\Gamma(1 / 2) k !(k+1 / 2)} \\
& =\frac{\pi R^{3 / 2} e^{-\gamma\left(a_{1}+8 b_{1}\right)\left(U_{K K}\right)}}{3 \sqrt{U_{K K}}}=\frac{\delta x_{4}}{4},
\end{aligned}
$$

where by equality in the first two lines we mean that we only keep the relevant terms which are non-vanishing in the limit $\epsilon \rightarrow 0$. Thus on adding the higher derivative corrections, the radius of $x_{4}$ changes, but the $\mathrm{D} 8$ and $\overline{D 8}$ branes continue to be at antipodal points on the $x_{4}$ circle. 


\section{Estimating the corrections to (axial) vector meson masses}

Having discussed the flavor brane configuration, we now consider the effect of the higher derivative corrections on the masses of the (axial) vector mesons in holographic QCD. These vector mesons are obtained from the fluctuations of the gauge fields on the D8 brane world volume in the background geometry. In fact, the fluctuations along the directions $x_{\mu}$ yield the vector mesons, while the fluctuations along $U$ yield the pions. Expanding the DBI action to quadratic order in the gauge fields, we get that

$S_{D 8}=-\frac{T_{8}}{4} \int d^{4} x d U e^{\gamma\left(\hat{\phi}_{1} / 4+b_{1}+4 c_{1}\right)(U)} \operatorname{Tr}\left[\frac{R^{9 / 2}}{\sqrt{U f(U)}} \hat{F}_{\mu \nu}^{2}+2 e^{-2 \gamma b_{1}(U)} U^{5 / 2} R^{3 / 2} \sqrt{f(U)} \hat{F}^{\mu}{ }_{U} F_{\mu U}\right]$

where

$$
T_{8}=\frac{\operatorname{Vol}\left(S^{4}\right)}{(2 \pi)^{6} \alpha^{\prime 5 / 2} g_{s}}
$$

and $\hat{F}$ means that the four dimensional indices are raised with the Minkowski metric.

We focus only on the vector mesons and drop the terms involving the pions. Thus defining

$$
A_{\mu}(x, U)=\sum_{n=1}^{\infty} B_{\mu}^{(n)}(x) \psi_{n}(U)
$$

we get that

$$
\begin{array}{r}
F_{\mu \nu}(x, U)=\sum_{n=1}^{\infty} F_{\mu \nu}^{(n)}(x) \psi_{n}(U), \\
F_{\mu U}(x, U)=-\sum_{n=1}^{\infty} B_{\mu}^{(n)}(x) \partial_{U} \psi_{n}(U),
\end{array}
$$

where $F_{\mu \nu}^{(n)}=\partial_{\mu} B_{\nu}^{(n)}-\partial_{\nu} B_{\mu}^{(n)}$. Thus we get that

$$
\begin{aligned}
S_{D 8}= & -\frac{T_{8}}{4} \int d^{4} x d U e^{\gamma\left(\hat{\phi}_{1} / 4+b_{1}+4 c_{1}\right)(U)} \sum_{m, n=1}^{\infty} \operatorname{Tr}\left[\frac{R^{9 / 2}}{\sqrt{U f(U)}} \hat{F}^{\mu \nu(m)}(x) F_{\mu \nu}^{(n)}(x) \psi_{m}(U) \psi_{n}(U)\right. \\
& \left.+2 e^{-2 \gamma b_{1}(U)} U^{5 / 2} R^{3 / 2} \sqrt{f(U)} \hat{B}^{\mu(m)}(x) B_{\mu}^{(n)}(x)\left(\partial_{U} \psi_{m}\right)(U)\left(\partial_{U} \psi_{n}\right)(U)\right] .
\end{aligned}
$$

Note that (30) is independent of $a_{1}$. Defining 


$$
Z^{2}=\frac{U^{3}}{U_{K K}^{3}}-1, \quad K(Z)=1+Z^{2},
$$

we get that

$$
\begin{aligned}
S_{D 8}= & -\hat{T}_{8} \int d^{4} x d Z e^{\gamma\left(\hat{\phi}_{1} / 4+b_{1}+4 c_{1}\right)(Z)} \sum_{m, n=1}^{\infty} \operatorname{Tr}\left[\frac{1}{4} K^{-1 / 3}(Z) \hat{F}^{\mu \nu(m)}(x) F_{\mu \nu}^{(n)}(x) \psi_{m}(Z) \psi_{n}(Z)\right. \\
& \left.+\frac{9 U_{K K}}{8 R^{3}} e^{-2 \gamma b_{1}(Z)} K(Z) \hat{B}^{\mu(m)}(x) B_{\mu}^{(n)}(x)\left(\partial_{Z} \psi_{m}\right)(Z)\left(\partial_{Z} \psi_{n}\right)(Z)\right]
\end{aligned}
$$

where

$$
\hat{T}_{8}=\frac{2 R^{9 / 2} \sqrt{U_{K K}} \operatorname{Vol}\left(S^{4}\right)}{3(2 \pi)^{6} \alpha^{\prime 5 / 2} g_{s}} .
$$

Canonical normalization of the kinetic term in (32) leads to

$$
\hat{T}_{8} \int d Z e^{\gamma\left(\hat{\phi}_{1} / 4+b_{1}+4 c_{1}\right)(Z)} K^{-1 / 3}(Z) \psi_{m}(Z) \psi_{n}(Z)=\delta_{m, n},
$$

while canonical normalization of the mass term gives

$$
\hat{T}_{8} \int d Z e^{\gamma\left(\hat{\phi}_{1} / 4-b_{1}+4 c_{1}\right)(Z)} K(Z)\left(\partial_{Z} \psi_{m}\right)(Z)\left(\partial_{Z} \psi_{n}\right)(Z)=\lambda_{n} \delta_{m, n},
$$

where the mass is given by $m_{n}^{2}=\left(9 U_{K K} \lambda_{n}\right) /\left(4 R^{3}\right)$. Thus (32) reduces to

$$
S_{D 8}=-\int d^{4} x \sum_{m=1}^{\infty} \operatorname{Tr}\left[\frac{1}{4} \hat{F}^{\mu \nu(n)} F_{\mu \nu}^{(n)}+\frac{m_{n}^{2}}{2} \hat{B}^{\mu(n)} B_{\mu}^{(n)}\right] .
$$

From (34) and (35) we obtain the eigenvalue equation for $\psi_{n}$

$$
e^{-\gamma\left(\hat{\phi}_{1} / 4+b_{1}+4 c_{1}\right)} K^{1 / 3} \partial_{Z}\left[e^{\gamma\left(\hat{\phi}_{1} / 4-b_{1}+4 c_{1}\right)} K\left(\partial_{Z} \psi_{n}\right)\right]=-\lambda_{n} \psi_{n}
$$

Now because the perturbations $\hat{\phi}_{1}, b_{1}$ and $c_{1}$ are functions of $Z^{2}$, note that the action (32) is invariant under $\left(x^{\mu}, Z\right) \rightarrow\left(-x^{\mu},-Z\right)$, which has the interpretation of space time parity [3]. Thus from (37) we see that $\psi_{n}$ has definite parity under $Z \rightarrow-Z$. The aim is to construct $\psi_{n}$ which has even (odd) parity for $n$ odd (even). Thus $B_{\mu}^{(n)}$ is a vector (axial vector) if $n$ is odd (even). Under charge conjugation, $B_{\mu}^{(n)}$ is even (odd) if $n$ is even (odd) [3]. We shall focus on the lowest lying modes with $n=1,2$ which have $(C, P)=(-,-)$ and $(+,+)$ respectively. In holographic QCD, these modes are to be identified with the $\rho$ meson and the $a_{1}(1260)$ meson respectively. 
In order to find the correction to the vector meson masses due to the higher derivative corrections, we write

$$
\begin{gathered}
\psi_{n}=\psi_{n}^{(0)}+\frac{\gamma}{\sqrt{R^{9} U_{K K}^{3}}} \psi_{n}^{(1)}, \\
\lambda_{n}=\lambda_{n}^{(0)}+\frac{\gamma}{\sqrt{R^{9} U_{K K}^{3}}} \lambda_{n}^{(1)},
\end{gathered}
$$

where $\psi_{n}^{(0)}$ and $\lambda_{n}^{(0)}$ are the supergravity expressions, while $\psi_{n}^{(1)}$ and $\lambda_{n}^{(1)}$ are the higher derivative corrections.

Using first order perturbation theory, (34), and (37), we get that

$$
\lambda_{n}^{(1)}=-\hat{T}_{8} \int d Z \psi_{n}^{(0)}(Z) \hat{H}_{n}(Z) \psi_{n}^{(0)}(Z)
$$

where

$$
\begin{aligned}
\hat{H}_{n}(Z) & =K\left(\frac{\hat{\phi}_{1}^{\prime}}{4}-b_{1}^{\prime}+4 c_{1}^{\prime}\right) \frac{\partial}{\partial Z}-2 b_{1}\left(2 Z \frac{\partial}{\partial Z}+K \frac{\partial^{2}}{\partial Z^{2}}\right) \\
& =K\left(\frac{\hat{\phi}_{1}^{\prime}}{4}-b_{1}^{\prime}+4 c_{1}^{\prime}\right) \frac{\partial}{\partial Z}+2 \lambda_{n}^{(0)} b_{1} K^{-1 / 3},
\end{aligned}
$$

where we have used the $O(1)$ relation from (37)

$$
K^{1 / 3}\left(K \partial_{Z}^{2} \psi_{n}^{(0)}+2 Z \partial_{Z} \psi_{n}^{(0)}\right)=-\lambda_{n}^{(0)} \psi_{n}^{(0)} .
$$

Integrating (39) by parts, one can also express $\lambda_{n}^{(1)}$ as

$$
\lambda_{n}^{(1)}=\hat{T}_{8} \int d Z K\left(\frac{\hat{\phi}_{1}}{4}-b_{1}+4 c_{1}\right)\left(\partial_{Z} \psi_{n}^{(0)}\right)^{2}-\lambda_{n}^{(0)} \hat{T}_{8} \int d Z K^{-1 / 3}\left(\frac{\hat{\phi}_{1}}{4}+b_{1}+4 c_{1}\right)\left(\psi_{n}^{(0)}\right)^{2}
$$

In (39) and the equations that follow, we have removed an overall factor of $1 / \sqrt{R^{9} U_{K K}^{3}}$ from $\hat{\phi}_{1}, b_{1}$ and $c_{1}$ for notational simplicity. Now it is difficult to calculate $\lambda_{n}^{(1)}$ from (39) (or (42)) exactly because the unperturbed wavefunction $\psi_{n}^{(0)}(Z)$ is not known exactly. Also although we have recursion relations for the coefficients describing the perturbed geometry, we do not have closed form expressions for them. So we will make a very rough estimate of the correction to the meson masses, which we turn to now.

Sakai and Sugimoto considered normalizable wavefunctions satisfying the Schrodinger equation (41), and the normalization condition (35), and obtained $\lambda_{n}^{(0)}$ numerically using the shooting technique. They obtained 


$$
\lambda_{1}^{(0)} \approx 0.67^{(-,-)}, \quad \lambda_{2}^{(0)} \approx 1.6^{(+,+)}
$$

for the two lightest modes that satisfy (41). Now for low values of $n$, these normalizable wave functions must be concentrated around $Z=0$, while they spread out more and more to larger values of $Z$ as $n$ increases. Since we will focus on the two lowest lying normalizable eigenstates of (41), the correction to the meson masses given by (39) (or (42)) should receive the maximum contribution from the neighbourhood of $Z=0$ in the integral. Thus in order to make a rough estimate of $\lambda_{n}^{(1)}$, we shall focus on this region only. Of course, as larger and larger values of $Z$ are considered, the approximation gets better and better.

So in the various expressions, we focus on the region of integration around $Z=0$. In fact, we shall make the crudest approximation, and restrict ourselves to terms only upto $O(Z)$ in the various expressions. Now it is easy to construct an approximate normalized wavefunction which solves (41) at small $Z$. Keeping terms only upto $O(Z)$, (41) reduces to

$$
\partial_{Z}^{2} \chi_{n}(Z) \approx\left(1-\lambda_{n}^{(0)}\right) \chi_{n}(Z)
$$

where $\psi_{n}^{(0)}(Z)=e^{-Z^{2} / 2} \chi_{n}(Z)$. Thus the approximate normalized wave functions for the two lowest modes are given by

$$
\psi_{1}^{(0)}(Z) \approx \frac{0.69}{\sqrt{\hat{T}_{8}}} e^{-Z^{2} / 2} \cosh \left(\frac{Z}{\sqrt{3}}\right)
$$

and

$$
\psi_{2}^{(0)}(Z) \approx \frac{1.58}{\sqrt{\hat{T}_{8}}} e^{-Z^{2} / 2} \sin \left(\sqrt{\frac{3}{5}} Z\right) .
$$

In order to estimate $\lambda_{n}^{(1)}$ at this order, we also need approximate expressions for $\hat{\phi}_{1}(Z), b_{1}(Z)$ and $c_{1}(Z)$. Because $\hat{\phi}_{1}(Z), b_{1}(Z)$ and $c_{1}(Z)$ are functions of $Z^{2}$ only, and we are restricting to terms of $O(Z)$, we can replace $\hat{\phi}_{1}(Z), b_{1}(Z)$ and $c_{1}(Z)$ by the constant modes $\hat{\phi}^{\star}, b^{\star}$ and $c^{\star}$ respectively, where 5

$$
\hat{\phi}_{1}(Z)=\hat{\phi}^{\star}+O\left(Z^{2}\right), \quad b_{1}(Z)=b^{\star}+O\left(Z^{2}\right), \quad c_{1}(Z)=c^{\star}+O\left(Z^{2}\right) .
$$

\footnotetext{
${ }^{5}$ In fact, we can look at the system of equations (69) at small $Z$, keeping terms only to $O(Z)$. Noting from the exact expressions for the perturbations in terms of $\eta$ that their behavior is similar at small $Z$, one can solve them directly, and obtain the solutions for $\hat{\phi}_{1}(Z), b_{1}(Z)$ and $c_{1}(Z)$ as described above. Note that the $Z$ dependence of the perturbations obtained by solving (69) at small $Z$, is very different from that in the wave functions (45) and (46) obtained by solving (41) at small $Z$.
} 
Thus using (34) and (35), from (39) (or (42)), we get that

$$
\lambda_{n}^{(1)} \approx-2 \lambda_{n}^{(0)} b^{\star}
$$

Thus at this order

$$
\frac{\delta m_{2}^{2}}{\delta m_{1}^{2}} \approx \frac{\lambda_{2}^{(0)}}{\lambda_{1}^{(0)}} \approx 2.4
$$

To obtain the masses, we estimate $b^{\star}$ using (69) directly. Setting $a_{1}(Z)=a^{\star}, b_{1}(Z)=b^{\star}$ and $c_{1}(Z)=c^{\star}$ and equating terms of $O(1)$, we get that 6

$$
b^{\star} \approx-20.6, \quad c^{\star} \approx-9.2 .
$$

Note that the zero mode $a^{\star}$ is undetermined by the equations.

In order to get better estimates, one has to keep terms at higher orders in $Z$, and solve for the wavefunction, as well as the metric and dilaton perturbations using (69) and (41). The $Z$ dependence of these quantities is going to be different, and the values of the various coefficients (for example, the constant terms in $b_{1}$ and $c_{1}$ ) are going to change too. However, on including the various contributions, $\lambda_{n}^{(1)}$ should not change by a large amount as the low lying states are localized around $Z=0$. It would be interesting to include higher powers of $Z$ in this analysis, and try to get a better estimate. Presumably keeping a reasonably small number of terms in the expansion in $Z$ will make the estimates converge to a sharp value of $\lambda_{n}^{(1)}$. Using our rough estimates, we get that

$$
\begin{aligned}
\frac{\delta m_{1}^{2}}{M_{K K}^{2}} & \approx 112\left(g_{Y M}^{2} N_{c}\right)^{-3} \\
\frac{\delta m_{2}^{2}}{M_{K K}^{2}} & \approx 267\left(g_{Y M}^{2} N_{c}\right)^{-3},
\end{aligned}
$$

where $M_{K K} \equiv 2 \pi /\left.\delta x_{4}\right|_{(\gamma=0)}=\left(3 \sqrt{U_{K K}}\right) /\left(2 R^{3 / 2}\right)$, and we have used the relations [7]

$$
R^{3}=\frac{g_{Y M}^{2} N_{c} \alpha^{\prime}}{2 M_{K K}}, \quad U_{K K}=\frac{2 g_{Y M}^{2} N_{c} M_{K K} \alpha^{\prime}}{9} .
$$

Thus at the level of the approximations we have made, we see that the masses of the two lightest (axial) vector mesons increase from their supergravity values. In fact, using

\footnotetext{
${ }^{6}$ Of the three resulting equations from (69), only two are linearly independent: the first two equations are the same.
} 
gauge/gravity duality techniques to calculate heavy quark potentials, one obtains that $g_{Y M}^{2} N_{c} \approx 1$ (see [31], for example). Thus our rough estimates give us that

$$
\frac{\delta m_{1}^{2}}{M_{K K}^{2}} \approx 112, \quad \frac{\delta m_{2}^{2}}{M_{K K}^{2}} \approx 267 .
$$

Trying to analyze the effect of the higher derivative corrections in other applications of holographic QCD is an important problem in general. It would be nice to have exact expressions for the metric and dilaton perturbations, as that will make calculations more concrete and predictive. In order to make precise quantitative predictions in holographic QCD due to corrections to supergravity at $O\left(\alpha^{\prime 3}\right)$, it is also important to understand the detailed structure of the $\mathcal{R}^{4}$ supermultiplet in type IIA string theory.

\section{Acknowledgements}

I would like to thank I. Klebanov, J. Maldacena, A. Maloney, D. Mateos, and S. Sugimoto for useful comments. I am particularly thankful to A. Maharana for many useful discussions, and for technical assistance in computing $\hat{W}$. The work of A. B. is supported by NSF Grant No. PHY-0503584 and the William D. Loughlin membership.

\section{$5 \quad$ Appendix}

In this appendix, we describe the construction of the perturbed metric and the dilaton due to the higher derivative corrections.

\section{A Obtaining the perturbed metric in the Einstein frame}

The aim is to first solve (11) to leading order in $\gamma$ and find the perturbed metric in the Einstein frame. Considering (11) at $O(\gamma)$, we get that

$$
S_{\text {Einstein }}^{O(\gamma)}=S_{0}^{\gamma}+S_{1}^{\gamma},
$$

where $S_{1}^{\gamma}$ contains $\phi_{1}$, and $S_{0}^{\gamma}$ is independent of it. Thus

$$
S_{0}^{\gamma}=V \int_{U_{K K}}^{\infty} d U \sqrt{\hat{\hat{g}}}\left[\hat{R}-\frac{9}{32 U^{2} H^{2} P^{2}}-\frac{g_{s}^{2} Q^{2}}{2 L^{8}}\left(\frac{U}{R}\right)^{3 / 8}+\gamma\left(\frac{U}{R}\right)^{-9 / 8} \hat{W}\right],
$$

and

$$
S_{1}^{\gamma}=V \int_{U_{K K}}^{\infty} d U \sqrt{\tilde{\hat{g}}}\left[-\frac{3 \partial_{U} \phi_{1}}{4 U H^{2} P^{2}}-\frac{g_{s}^{2} Q^{2}}{4 L^{8}}\left(\frac{U}{R}\right)^{3 / 8} \phi_{1}\right]
$$


Since $\phi_{1} \sim O(\gamma)$, we can replace the other fields in (56) by their supergravity values. Thus we get

$$
S_{1}^{\gamma}=V \int_{U_{K K}}^{\infty} d U\left[-\frac{3 f(U) U^{3} \partial_{U} \phi_{1}}{4}-\frac{g_{s}^{2} Q^{2} U^{2} \phi_{1}}{4 R^{6}}\right],
$$

which vanishes on integrating by parts the first term, and using (2) and (44). So in order to find the metric perturbation to $O(\gamma)$, we only need to consider (55). Note that the dilaton perturbation to leading order is undetermined at $O(\gamma)$ in the perturbative expansion.

To evaluate (55), we use the parametrizations

$$
H(U)=\left(\frac{U}{R}\right)^{9 / 16}, \quad K(U)=e^{a(U)+\lambda b(U)}, \quad P(U)=e^{b(U)}, \quad L(U)=e^{c(U)} U\left(\frac{R}{U}\right)^{15 / 16}
$$

where $\lambda$ is a constant, which we now fix to simplify calculations. So in (158), we have $c(U) \sim O(\gamma)$. Using (158), we calculate $\sqrt{\hat{\hat{g}}} \hat{R}$ and get

$$
\begin{aligned}
\sqrt{\tilde{\hat{g}} \hat{R}=} & -\frac{e^{a+(\lambda-1) b+4 c} \sqrt{U R}}{8}\left[\frac{21 R}{4}-96 U^{2} R\left(c^{\prime}\right)^{2}-96 e^{2(b-c)} U\left(\frac{U}{R}\right)^{2}\right. \\
& \left.+5 U R a^{\prime}+5(\lambda-9) U R b^{\prime}-12 U R c^{\prime}-64 U^{2} R c^{\prime}\left(a^{\prime}+\lambda b^{\prime}\right)\right] \\
& -2 R^{3 / 2} \frac{d}{d U}\left[U^{5 / 2}\left(a^{\prime}+\lambda b^{\prime}+4 c^{\prime}+\frac{1}{4 U}\right) e^{a+(\lambda-1) b+4 c}\right] .
\end{aligned}
$$

Note that for $\lambda=9$, the coefficient of the $U R b^{\prime}$ term vanishes, and this is the value we choose. Thus (55) yields

$$
S_{0}^{\gamma}=V \int_{U_{K K}}^{\infty} d U\left[l\left(a, a^{\prime}, b, b^{\prime}, c, c^{\prime}\right)+\gamma w\left(a, a^{\prime}, a^{\prime \prime}, b, b^{\prime}, b^{\prime \prime}, c, c^{\prime}, c^{\prime \prime}\right)\right]
$$

where

$$
\begin{aligned}
l\left(a, a^{\prime}, b, b^{\prime}, c, c^{\prime}\right)= & -\frac{e^{a+8 b+4 c} \sqrt{U R}}{8}\left[\frac{21 R}{4}-96 U^{2} R\left(c^{\prime}\right)^{2}-96 e^{2(b-c)} U\left(\frac{U}{R}\right)^{2}\right. \\
& \left.+5 U R a^{\prime}-12 U R c^{\prime}-64 U^{2} R c^{\prime}\left(a^{\prime}+9 b^{\prime}\right)\right] \\
& -\frac{9}{32} U^{2} e^{a+8 b+4 c}\left[\left(\frac{R}{U}\right)^{3 / 2}+16\left(\frac{U}{R}\right)^{3 / 2} e^{2 b-8 c}\right],
\end{aligned}
$$

where we have dropped the total derivative, and

$$
w\left(a, a^{\prime}, a^{\prime \prime}, b, b^{\prime}, b^{\prime \prime}, c, c^{\prime}, c^{\prime \prime}\right)=U(U R)^{3 / 2} e^{a+10 b+4 c} \hat{W}\left(a, a^{\prime}, a^{\prime \prime}, b, b^{\prime}, b^{\prime \prime}, c, c^{\prime}, c^{\prime \prime}\right) .
$$


So we need to solve the Euler-Lagrange equations of motion arising from (60) which are given by

$$
\frac{\partial l}{\partial \xi_{i}}-\frac{d}{d U}\left(\frac{\partial l}{\partial \xi_{i}^{\prime}}\right)=-\gamma\left[\frac{\partial w}{\partial \xi_{i}}-\frac{d}{d U}\left(\frac{\partial w}{\partial \xi_{i}^{\prime}}\right)+\frac{d^{2}}{d U^{2}}\left(\frac{\partial w}{\partial \xi_{i}^{\prime \prime}}\right)\right]
$$

where $\xi_{i}=a, b, c$. The equations of motion for $a, b$ and $c$ are given by

$$
\begin{aligned}
& \left(\frac{U}{R}\right)^{3 / 2}\left[3 e^{2 b} U^{2}\left(8 e^{6 c}-3\right)-2 R^{3} e^{8 c}\left(8 U c^{\prime \prime}-b^{\prime}\left(5+8 U c^{\prime}\right)+4 c^{\prime}\left(4+5 U c^{\prime}\right)\right)\right] \\
& =-\frac{2 \gamma}{e^{a+8 b-4 c}}\left[\frac{\partial w}{\partial a}-\frac{d}{d U}\left(\frac{\partial w}{\partial a^{\prime}}\right)+\frac{d^{2}}{d U^{2}}\left(\frac{\partial w}{\partial a^{\prime \prime}}\right)\right], \\
& \sqrt{\frac{U}{R}}\left[R^{2} e^{8 c}\left(15 R+2 U R a^{\prime}\left(5+8 U c^{\prime}\right)+48 U R c^{\prime}\left(7+8 U c^{\prime}\right)+144 U^{2} R c^{\prime \prime}\right)\right. \\
& \left.+10 e^{2 b}\left(9-24 e^{6 c}\right) U^{3}\right]=\frac{2 R \gamma}{e^{a+8 b-4 c}}\left[\frac{\partial w}{\partial b}-\frac{d}{d U}\left(\frac{\partial w}{\partial b^{\prime}}\right)+\frac{d^{2}}{d U^{2}}\left(\frac{\partial w}{\partial b^{\prime \prime}}\right)\right], \\
& \sqrt{\frac{U}{R}}\left[6 U^{3} e^{2 b}\left(3+4 e^{6 c}\right)-2 R^{3} e^{8 c}\left\{3+2 U\left(2 U a^{\prime 2}+15 c^{\prime}+2 a^{\prime}\left(3+17 U b^{\prime}+3 U c^{\prime}\right)\right.\right.\right. \\
& \left.\left.\left.+2\left[72 U b^{\prime 2}+24 b^{\prime}\left(1+U c^{\prime}\right)+U\left(6 c^{2}+a^{\prime \prime}+9 b^{\prime \prime}+3 c^{\prime \prime}\right)\right]\right)\right\}\right] \\
& =-\frac{R \gamma}{e^{a+8 b-4 c}}\left[\frac{\partial w}{\partial c}-\frac{d}{d U}\left(\frac{\partial w}{\partial c^{\prime}}\right)+\frac{d^{2}}{d U^{2}}\left(\frac{\partial w}{\partial c^{\prime \prime}}\right)\right],
\end{aligned}
$$

respectively. We now expand (64) to $O(\gamma)$. Since the right hand side is already of $O(\gamma)$, we simply substitute the values in the supergravity solution. Defining

$$
\frac{U_{K K}}{U} \equiv \eta
$$

the relevant expressions are

$$
\begin{aligned}
& \frac{\partial w}{\partial a}-\frac{d}{d U}\left(\frac{\partial w}{\partial a^{\prime}}\right)+\frac{d^{2}}{d U^{2}}\left(\frac{\partial w}{\partial a^{\prime \prime}}\right) \\
= & \frac{3 \sqrt{U R}}{512 R^{5}}\left[\frac{729}{2}-2106 \eta^{3}+\frac{22221}{2} \eta^{6}-474174 \eta^{9}+\frac{1072869}{2} \eta^{12}\right], \\
& \frac{\partial w}{\partial b}-\frac{d}{d U}\left(\frac{\partial w}{\partial b^{\prime}}\right)+\frac{d^{2}}{d U^{2}}\left(\frac{\partial w}{\partial b^{\prime \prime}}\right) \\
= & \frac{3 \sqrt{U R}}{512 R^{5}}\left[3753-19008 \eta^{3}+105831 \eta^{6}-4273602 \eta^{9}+4899321 \eta^{12}\right], \\
& \frac{\partial w}{\partial c}-\frac{d}{d U}\left(\frac{\partial w}{\partial c^{\prime}}\right)+\frac{d^{2}}{d U^{2}}\left(\frac{\partial w}{\partial c^{\prime \prime}}\right) \\
= & -\frac{3 \sqrt{U R}}{256 R^{5}}\left[945+216 \eta^{3}-20106 \eta^{6}+37581 \eta^{9}-16569 \eta^{12}\right] .
\end{aligned}
$$


On the left hand side of (64), the contributions due to terms of $O(1)$ vanish, and the $O(\gamma)$ terms are the leading effects. Defining the order $\gamma$ perturbations to the metric by

$$
\begin{aligned}
a(U) & =-\frac{27}{2} \ln \left(\frac{R}{U}\right)+5 \ln f(U)+\gamma a_{1}(U), \\
b(U) & =\frac{3}{2} \ln \left(\frac{R}{U}\right)-\frac{1}{2} \ln f(U)+\gamma b_{1}(U), \\
c(U) & =\gamma c_{1}(U),
\end{aligned}
$$

where $a_{1}, b_{1}$, and $c_{1}$ are $O(1)$, substituting them into (64), and equating terms of $O(\gamma)$ we get the equations satisfied by the metric perturbations. Defining the dimensionless variables

$$
A_{1}=\left(R^{9} U_{K K}^{3}\right)^{1 / 2} a_{1}, \quad B_{1}=\left(R^{9} U_{K K}^{3}\right)^{1 / 2} b_{1}, \quad C_{1}=\left(R^{9} U_{K K}^{3}\right)^{1 / 2} c_{1},
$$

and changing coordinates to $\eta$, from (64) we get that

$$
\begin{aligned}
& -8\left(1-\eta^{3}\right) C_{1}^{\prime \prime}+\frac{12 C_{1}^{\prime}}{\eta}-\frac{5\left(1-\eta^{3}\right) B_{1}^{\prime}}{\eta}+\frac{15 B_{1}+12 C_{1}}{\eta^{2}} \\
= & -\frac{3}{512 \sqrt{\eta}}\left[\frac{729}{2}-2106 \eta^{3}+\frac{22221}{2} \eta^{6}-474174 \eta^{9}+\frac{1072869}{2} \eta^{12}\right] \equiv \frac{f_{1}\left(\eta^{3}\right)}{\sqrt{\eta}} \\
& 12\left(1-\eta^{3}\right) C_{1}^{\prime \prime}+\frac{2\left(\eta^{3}-11\right) C_{1}^{\prime}}{\eta}-\frac{5\left(1-\eta^{3}\right) A_{1}^{\prime}}{6 \eta}-\frac{5\left(5 B_{1}+4 C_{1}\right)}{\eta^{2}} \\
= & \frac{1}{1024 \sqrt{\eta}}\left[3753-19008 \eta^{3}+105831 \eta^{6}-4273602 \eta^{9}+4899321 \eta^{12}\right] \equiv \frac{f_{2}\left(\eta^{3}\right)}{\sqrt{\eta}}, \\
& -2\left(1-\eta^{3}\right)\left(A_{1}^{\prime \prime}+9 B_{1}^{\prime \prime}+3 C_{1}^{\prime \prime}\right)+\frac{3\left(7 B_{1}-16 C_{1}\right)}{\eta^{2}} \\
& +\frac{\left(4 \eta^{3}+5\right) A_{1}^{\prime}}{\eta}+\frac{39\left(\eta^{3}+1\right) B_{1}^{\prime}}{\eta}+\frac{6\left(\eta^{3}+2\right) C_{1}^{\prime}}{\eta} \\
= & \frac{3}{1024 \sqrt{\eta}}\left[945+216 \eta^{3}-20106 \eta^{6}+37581 \eta^{9}-16569 \eta^{12}\right] \equiv \frac{f_{3}\left(\eta^{3}\right)}{\sqrt{\eta}} .
\end{aligned}
$$

From the equations (69), it follows that $a_{1}$ has a zero mode given by $a_{1}=$ const, which is not fixed by the equations of motion. We shall fix its value shortly.

Ignoring the issue of the zero mode of $A_{1}$ for the time being, we now solve the equations (69) in order to find the metric perturbations in the Einstein frame. We find it convenient to further redefine variables 


$$
A_{1}=\eta^{3 / 2} \mathcal{A}_{1}, \quad B_{1}=\eta^{3 / 2} \mathcal{B}_{1}, \quad C_{1}=\eta^{3 / 2} \mathcal{C}_{1},
$$

so that the metric perturbations are given by

$$
a_{1}(U)=\frac{\mathcal{A}_{1}(U)}{\sqrt{R^{9} U^{3}}}, \quad b_{1}(U)=\frac{\mathcal{B}_{1}(U)}{\sqrt{R^{9} U^{3}}}, \quad c_{1}(U)=\frac{\mathcal{C}_{1}(U)}{\sqrt{R^{9} U^{3}}},
$$

Using this, we see that equations (69) reduce to

$$
\begin{aligned}
& -8\left(1-\eta^{3}\right) \eta^{2} \mathcal{C}_{1}^{\prime \prime}+12\left(2 \eta^{3}-1\right) \eta \mathcal{C}_{1}^{\prime}-5\left(1-\eta^{3}\right) \eta \mathcal{B}_{1}^{\prime} \\
& +\frac{15\left(1+\eta^{3}\right)}{2} \mathcal{B}_{1}+6\left(\eta^{3}+4\right) \mathcal{C}_{1}=f_{1}\left(\eta^{3}\right), \\
& 12\left(1-\eta^{3}\right) \eta^{2} \mathcal{C}_{1}^{\prime \prime}+2\left(7-17 \eta^{3}\right) \eta \mathcal{C}_{1}^{\prime}-\frac{5\left(1-\eta^{3}\right)}{6} \eta \mathcal{A}_{1}^{\prime} \\
& -\frac{5\left(1-\eta^{3}\right)}{4} \mathcal{A}_{1}-25 \mathcal{B}_{1}-2\left(22+3 \eta^{3}\right) \mathcal{C}_{1}=f_{2}\left(\eta^{3}\right), \\
& -2\left(1-\eta^{3}\right) \eta^{2}\left(\mathcal{A}_{1}^{\prime \prime}+9 \mathcal{B}_{1}^{\prime \prime}+3 \mathcal{C}_{1}^{\prime \prime}\right) \\
& +\left(10 \eta^{3}-1\right) \eta \mathcal{A}_{1}^{\prime}+3\left(31 \eta^{3}-5\right) \eta \mathcal{B}_{1}^{\prime}+6\left(4 \eta^{3}-1\right) \eta \mathcal{C}_{1}^{\prime} \\
& +3\left(\frac{5 \eta^{3}}{2}+2\right) \mathcal{A}_{1}+6\left(12 \eta^{3}+11\right) \mathcal{B}_{1}+\frac{3\left(9 \eta^{3}-23\right)}{2} \mathcal{C}_{1}=f_{3}\left(\eta^{3}\right) .
\end{aligned}
$$

Though the system of equations (72) looks complicated, it is easy to see that they are solved by sums of powers of harmonic functions $\eta^{3}$. So we make the ansatz

$$
\mathcal{A}_{1}(\eta)=\sum_{k=0}^{\infty} \frac{\hat{a}_{3 k}}{512} \eta^{3 k}, \quad \mathcal{B}_{1}(\eta)=\sum_{k=0}^{\infty} \frac{\hat{b}_{3 k}}{512} \eta^{3 k}, \quad \mathcal{C}_{1}(\eta)=\sum_{k=0}^{\infty} \frac{\hat{c}_{3 k}}{512} \eta^{3 k},
$$

where $\hat{a}_{3 k}, \hat{b}_{3 k}$ and $\hat{c}_{3 k}$ are numbers. Demanding regularity of the solution as $U_{K K} \rightarrow 0$, we do not have negative values of $k$ in (73)). Thus including the zero mode, $a_{1}(U)$ is given by

$$
a_{1}(U)=\text { const }+\frac{1}{\sqrt{R^{9} U^{3}}} \sum_{k=0}^{\infty} \frac{\hat{a}_{3 k}}{512}\left(\frac{U_{K K}}{U}\right)^{3 k} .
$$

In the supersymmetric limit $U_{K K} \rightarrow 0, a_{1}(U)$ is given by

$$
a_{1}(U)=\text { const }+\frac{\hat{a}_{0}}{512 \sqrt{R^{9} U^{3}}} .
$$

We set the zero mode to zero so that the $x_{4}$ coordinate is canonically normalized [17]. Solving (72) boils down to solving a system of coupled difference equations obtained by equating terms involving the same powers of $\eta$. The equations are 


$$
\begin{aligned}
\frac{5 \hat{b}_{0}}{2}+8 \hat{c}_{0} & =-\frac{729}{2} \\
\frac{5 \hat{a}_{0}}{4}+25 \hat{b}_{0}+44 \hat{c}_{0} & =-\frac{3753}{2} \\
2 \hat{a}_{0}+22 \hat{b}_{0}-\frac{23 \hat{c}_{0}}{2} & =\frac{945}{2}
\end{aligned}
$$

and

$$
\begin{aligned}
& -4\left(k-\frac{1}{2}\right)\left(2(3 k+2) \hat{c}_{3 k}-(6 k-5) \hat{c}_{3(k-1)}\right)+\frac{5(1-2 k)}{2}\left(\hat{b}_{3 k}-\hat{b}_{3(k-1)}\right) \\
& =2106 \delta_{k, 1}-\frac{22221}{2} \delta_{k, 2}+474174 \delta_{k, 3}-\frac{1072869}{2} \delta_{k, 4}, \\
& -\frac{5(2 k+1)}{4} \hat{a}_{3 k}+\frac{5(2 k-1)}{4} \hat{a}_{3(k-1)}+6\left(18 k^{2}+k-\frac{22}{3}\right) \hat{c}_{3 k}-6\left(18 k^{2}-25 k+8\right) \hat{c}_{3(k-1)} \\
& -25 \hat{b}_{3 k}=-9504 \delta_{k, 1}+\frac{105831}{2} \delta_{k, 2}-2136801 \delta_{k, 3}+\frac{4899321}{2} \delta_{k, 4}, \\
& \left(-6 k^{2}+k+2\right) \hat{a}_{3 k}+\left(6 k^{2}-4 k+\frac{1}{2}\right) \hat{a}_{3(k-1)}+\left(-54 k^{2}+3 k+22\right) \hat{b}_{3 k} \\
& +3\left(18 k^{2}-11 k+1\right) \hat{b}_{3(k-1)}-\left(18 k^{2}+\frac{23}{2}\right) \hat{c}_{3 k}+9\left(2 k^{2}-2 k+\frac{1}{2}\right) \hat{c}_{3(k-1)} \\
& =108 \delta_{k, 1}-10053 \delta_{k, 2}+\frac{37581}{2} \delta_{k, 3}-\frac{16569}{2} \delta_{k, 4},
\end{aligned}
$$

for $k \geq 1$. These equations can be solved recursively and yield the solution

$$
\begin{aligned}
& \mathcal{A}_{1}(\eta)=\frac{1}{512}\left[\frac{2034}{5}+\frac{41106}{5} \eta^{3}-\frac{20491152}{175} \eta^{6}+\frac{5360956}{21} \eta^{9}-\frac{1116115426}{17325} \eta^{12}+\ldots\right] \\
& \mathcal{B}_{1}(\eta)=\frac{1}{512}\left[-\frac{169}{5}-845 \eta^{3}+\frac{454014}{35} \eta^{6}-\frac{129590014}{4725} \eta^{9}+\frac{391267183}{51975} \eta^{12}+\ldots\right] \\
& \mathcal{C}_{1}(\eta)=\frac{1}{512}\left[-35-\frac{37}{5} \eta^{3}-\frac{33843}{35} \eta^{6}-\frac{81526}{189} \eta^{9}-\frac{719803}{1485} \eta^{12}+\ldots\right]
\end{aligned}
$$

where $\ldots$ are the terms higher order in $\eta$, starting from $\eta^{15}$. We do not have a closed form expression for the metric perturbations. Thus, for example, we cannot determine the precise nature of the perturbations near $U=U_{K K}$. Note that the metric perturbations vanish as $U \rightarrow \infty$, i.e., far away from the color branes.

\section{B Obtaining the perturbed dilaton}

We next calculate the dilaton perturbation at $O(\gamma)$, for which we need to consider the action (11) at $O\left(\gamma^{2}\right)$. Including only the terms that depend on the dilaton, this gives 


$$
S_{\phi}^{\gamma^{2}}=S_{\phi_{2}}^{\gamma^{2}}+S_{\phi_{1}}^{\gamma^{2}}
$$

where

$$
S_{\phi_{2}}^{\gamma^{2}}=V \int_{U_{K K}}^{\infty} d U \sqrt{\tilde{\hat{g}}}\left[-\frac{3 \partial_{U} \phi_{2}}{4 U H^{2} P^{2}}-\frac{g_{s}^{2} Q^{2}}{4 L^{8}}\left(\frac{U}{R}\right)^{3 / 8} \phi_{2}\right],
$$

which depends on $\phi_{2}$, and

$$
\begin{array}{r}
S_{\phi_{1}}^{\gamma^{2}}=V \int_{U_{K K}}^{\infty} d U \sqrt{\hat{g}}\left[-\frac{3 \partial_{U} \phi_{1}}{4 U H^{2} P^{2}}-\frac{\left(\partial_{U} \phi_{1}\right)^{2}}{2 H^{2} P^{2}}-\frac{g_{s}^{2}}{4 \cdot 4 !}\left(\frac{U}{R}\right)^{3 / 8}\left(\phi_{1}+\frac{\phi_{1}^{2}}{4}\right) \hat{F}_{4}^{2}\right. \\
\left.-\frac{3}{2} \gamma\left(\frac{U}{R}\right)^{-9 / 8} \hat{W} \phi_{1}\right],
\end{array}
$$

which depends on $\phi_{1}$. In (80), $\phi_{2} \sim O\left(\gamma^{2}\right)$, and so we substitute the supergravity values of the metric and the four form flux. Thus $S_{\phi_{2}}^{\gamma^{2}}$ vanishes for the same reason as in (56). In (81), we substitute the supergravity values of the various fields in the terms involving $\phi_{1}^{2}$ and in the term involving $\hat{W} \phi_{1}$, while in the remaining terms we substitute the values of the fields at $O(\gamma)$. Now $\hat{W}$ for the supergravity metric (9) is given by

$$
\hat{W}=\frac{9\left(135 U^{12}+819 U^{6} U_{K K}^{6}-756 U^{3} U_{K K}^{9}+4739 U_{K K}^{12}\right)}{1024 U^{25 / 2} R^{15 / 2}} .
$$

Note that (82) does not vanish for $U_{K K}=0$, as the theory is not conformal. Thus defining $\phi_{1}=\gamma \hat{\phi}_{1}$, we get that

$$
\begin{aligned}
S_{\phi_{1}}^{\gamma^{2}}= & \gamma^{2} V \int_{U_{K K}}^{\infty} d U\left[-\frac{f(U) U^{4}\left(\partial_{U} \hat{\phi}_{1}\right)^{2}}{2}-\frac{9 U^{2} \hat{\phi}_{1}^{2}}{16}\right. \\
& -\frac{3 f(U) U^{3}}{4}\left(a_{1}+8 b_{1}+4 c_{1}\right)\left(\partial_{U} \hat{\phi}_{1}\right)-\frac{9 U^{2}}{4}\left(a_{1}+10 b_{1}-4 c_{1}\right) \hat{\phi}_{1} \\
& \left.-\frac{27\left(135 U^{12}+819 U^{6} U_{K K}^{6}-756 U^{3} U_{K K}^{9}+4739 U_{K K}^{12}\right) \hat{\phi}_{1}}{2048 U^{23 / 2} R^{9 / 2}}\right] .
\end{aligned}
$$

This leads to the equation of motion

$$
\begin{aligned}
& \left(1-\eta^{3}\right) \varphi_{1}^{\prime \prime}-\frac{\left(\eta^{3}+2\right)}{\eta} \varphi_{1}^{\prime}-\frac{9 \varphi_{1}}{8 \eta^{2}} \\
= & \frac{9}{4 \sqrt{\eta}}\left(\mathcal{A}_{1}+10 \mathcal{B}_{1}-4 \mathcal{C}_{1}\right)-\frac{9\left(\eta^{3}+1\right)}{8 \sqrt{\eta}}\left(\mathcal{A}_{1}+8 \mathcal{B}_{1}+4 \mathcal{C}_{1}\right)
\end{aligned}
$$




$$
\begin{aligned}
& +\frac{3\left(1-\eta^{3}\right) \sqrt{\eta}}{4}\left(\mathcal{A}_{1}^{\prime}+8 \mathcal{B}_{1}^{\prime}+4 \mathcal{C}_{1}^{\prime}\right) \\
& +\frac{27}{2048 \sqrt{\eta}}\left(135+819 \eta^{6}-756 \eta^{9}+4739 \eta^{12}\right),
\end{aligned}
$$

where $\varphi_{1}$ is the dimensionless dilaton perturbation defined by

$$
\varphi_{1}=\left(R^{9} U_{K K}^{3}\right)^{1 / 2} \hat{\phi}_{1},
$$

and all derivatives in (84) are with respect to $\eta$. Just like the metric perturbations, defining $\varphi_{1}=\eta^{3 / 2} \hat{\varphi}_{1}$, (84) leads to

$$
\begin{aligned}
& \left(1-\eta^{3}\right) \eta^{2} \hat{\varphi}_{1}^{\prime \prime}+\left(1-4 \eta^{3}\right) \eta \hat{\varphi}_{1}^{\prime}-\frac{9\left(\eta^{3}+\frac{3}{2}\right)}{4} \hat{\varphi}_{1} \\
= & \frac{9}{4}\left(\mathcal{A}_{1}+10 \mathcal{B}_{1}-4 \mathcal{C}_{1}\right)-\frac{9\left(\eta^{3}+1\right)}{8}\left(\mathcal{A}_{1}+8 \mathcal{B}_{1}+4 \mathcal{C}_{1}\right) \\
& +\frac{3\left(1-\eta^{3}\right) \eta}{4}\left(\mathcal{A}_{1}^{\prime}+8 \mathcal{B}_{1}^{\prime}+4 \mathcal{C}_{1}^{\prime}\right) \\
& +\frac{27}{2048}\left(135+819 \eta^{6}-756 \eta^{9}+4739 \eta^{12}\right) .
\end{aligned}
$$

which we solve by making the ansatz

$$
\hat{\varphi}_{1}(\eta)=\sum_{k=0}^{\infty} \frac{\tilde{\varphi}_{3 k}}{512} \eta^{3 k}
$$

The difference equations are

$$
\tilde{\varphi}_{0}=-\frac{\hat{a}_{0}}{3}-4 \hat{b}_{0}+4 \hat{c}_{0}-270
$$

and

$$
\begin{aligned}
& \left(k^{2}-\frac{3}{8}\right) \tilde{\varphi}_{3 k}-\left(k^{2}-k+\frac{1}{4}\right) \tilde{\varphi}_{3(k-1)} \\
= & \frac{k+\frac{1}{2}}{4} \hat{a}_{3 k}-\frac{k-\frac{1}{2}}{4} \hat{a}_{3(k-1)}+\left(2 k+\frac{3}{2}\right) \hat{b}_{3 k}-(2 k-1) \hat{b}_{3(k-1)} \\
& +\left(k-\frac{3}{2}\right) \hat{c}_{3 k}-\left(k-\frac{1}{2}\right) \hat{c}_{3(k-1)}+\frac{3}{4}\left(819 \delta_{k, 2}-756 \delta_{k, 3}+4739 \delta_{k, 4}\right),
\end{aligned}
$$

for $k \geq 1$. Thus on using (78), this leads to

$$
\hat{\varphi}_{1}(\eta)=\frac{1}{512}\left[-\frac{2052}{5}+\frac{216}{5} \eta^{3}-\frac{17172}{29} \eta^{6}+\frac{9086952}{3335} \eta^{9}+\frac{130277364}{416875} \eta^{12}+\ldots\right]
$$

where ... are terms of $O\left(\eta^{15}\right)$. 


\section{References}

[1] E. Witten, "Anti-de Sitter space, thermal phase transition, and confinement in gauge theories," Adv. Theor. Math. Phys. 2 (1998) 505-532, hep-th/9803131.

[2] A. Karch and E. Katz, "Adding flavor to AdS/CFT," JHEP 06 (2002) 043, hep-th/0205236.

[3] T. Sakai and S. Sugimoto, "Low energy hadron physics in holographic QCD," Prog. Theor. Phys. 113 (2005) 843-882, hep-th/0412141.

[4] T. Sakai and S. Sugimoto, "More on a holographic dual of QCD," Prog. Theor. Phys. 114 (2006) 1083-1118, hep-th/0507073.

[5] D. T. Son and M. A. Stephanov, "QCD and dimensional deconstruction," Phys. Rev. D69 (2004) 065020, hep-ph/0304182.

[6] J. Babington, J. Erdmenger, N. J. Evans, Z. Guralnik, and I. Kirsch, "Chiral symmetry breaking and pions in non-supersymmetric gauge / gravity duals," Phys. Rev. D69 (2004) 066007, hep-th/0306018.

[7] M. Kruczenski, D. Mateos, R. C. Myers, and D. J. Winters, "Towards a holographic dual of large-N(c) QCD," JHEP 05 (2004) 041, hep-th/0311270.

[8] O. Aharony, J. Sonnenschein, and S. Yankielowicz, "A holographic model of deconfinement and chiral symmetry restoration," Annals Phys. 322 (2007) 1420-1443, hep-th/0604161.

[9] D. Gepner and S. S. Pal, "Chiral symmetry breaking and restoration from holography," hep-th/0608229.

[10] E. Antonyan, J. A. Harvey, S. Jensen, and D. Kutasov, "NJL and QCD from string theory," hep-th/0604017.

[11] A. Parnachev and D. A. Sahakyan, "Chiral phase transition from string theory," Phys. Rev. Lett. 97 (2006) 111601, hep-th/0604173.

[12] Y.-h. Gao, W.-s. Xu, and D.-f. Zeng, "NGN, QCD(2) and chiral phase transition from string theory," JHEP 08 (2006) 018, hep-th/0605138. 
[13] E. Antonyan, J. A. Harvey, and D. Kutasov, "The Gross-Neveu model from string theory," hep-th/0608149.

[14] E. Antonyan, J. A. Harvey, and D. Kutasov, "Chiral symmetry breaking from intersecting D-branes," hep-th/0608177.

[15] A. Basu and A. Maharana, "Generalized Gross-Neveu models and chiral symmetry breaking from string theory," Phys. Rev. D75 (2007) 065005, hep-th/0610087.

[16] D. Bak and H.-U. Yee, "Separation of spontaneous chiral symmetry breaking and confinement via AdS/CFT correspondence," Phys. Rev. D71 (2005) 046003, hep-th/0412170.

[17] S. S. Gubser, I. R. Klebanov, and A. A. Tseytlin, "Coupling constant dependence in the thermodynamics of $\mathrm{N}=4$ supersymmetric Yang-Mills theory," Nucl. Phys. B534 (1998) 202-222, hep-th/9805156.

[18] J. Pawelczyk and S. Theisen, "AdS(5) x S(5) black hole metric at $O\left(\alpha^{\prime 3}\right)$," JHEP 09 (1998) 010, hep-th/9808126.

[19] M. M. Caldarelli and D. Klemm, "M-theory and stringy corrections to anti-de Sitter black holes and conformal field theories," Nucl. Phys. B555 (1999) 157-182, hep-th/9903078.

[20] S. de Haro, A. Sinkovics, and K. Skenderis, "A supersymmetric completion of the $R^{4}$ term in IIB supergravity," Phys. Rev. D67 (2003) 084010, hep-th/0210080.

[21] G. Policastro and D. Tsimpis, " $R^{4}$, purified," Class. Quant. Grav. 23 (2006) 4753, hep-th/0603165.

[22] G. T. Horowitz and A. Strominger, "Black strings and P-branes," Nucl. Phys. B360 (1991) 197-209.

[23] J. Polchinski, "String Theory, Volume 2: Superstring Theory and Beyond," Cambridge Monographs on Mathematical Physics. (1998).

[24] M. B. Green and J. H. Schwarz, "Supersymmetrical string theories," Phys. Lett. B109 (1982) 444-448.

[25] M. B. Green and J. H. Schwarz, "Supersymmetrical dual string theory. 3. Loops and renormalization," Nucl. Phys. B198 (1982) 441-460. 
[26] D. J. Gross and E. Witten, "Superstring Modifications of Einstein's Equations," Nucl. Phys. B277 (1986) 1.

[27] E. D'Hoker and D. H. Phong, "The geometry of string perturbation theory," Rev. Mod. Phys. 60 (1988) 917.

[28] M. T. Grisaru and D. Zanon, "Sigma model superstring corrections to the Einstein-Hilbert action," Phys. Lett. B177 (1986) 347.

[29] M. D. Freeman, C. N. Pope, M. F. Sohnius, and K. S. Stelle, "Higher order sigma model counterterms and the effective action for superstrings," Phys. Lett. B178 (1986) 199.

[30] Q.-H. Park and D. Zanon, "More on sigma model beta functions and low-energy effective actions," Phys. Rev. D35 (1987) 4038.

[31] O. Andreev and V. I. Zakharov, "Heavy-quark potentials and AdS/QCD," Phys. Rev. D74 (2006) 025023, hep-ph/0604204. 